\title{
Les Simuliidae (Diptera : Nematocera) de la Neste d'Aure (Pyrénées Centrales). I. Impact des aménagements hydroélectriques dans la haute vallée
}

\author{
M. Clergue-Gazeau ${ }^{1}$ \\ G. Gazagnes ${ }^{1}$
}

Mots clés : Simuliidac, eaux courantes, impact humain, Pyrénées.

Les auteurs présentent une liste de Simuliidae récol tées par dérive, en été et en hiver, dans un torrent de moyenne altitude : la Neste d'Aure 1100-1600 m (Hautes-Pyrénées).

La répartition des peuplements est analysée en fonction de huit stations de dérive, choisies entre l'amont et l'aval de trois retenues de prise d'eau. L'importance d'un débit réservé à l'aval d'une retenue est soulignée.

The Simuliidae (Diptera : Nematocera) of the Neste d'Aure (Central Pyrenees) I. The impact of hydroelectric schemes in the upper valley.

Keywords : Simuliidae, running waters, human impact, Pyrenees.

The authors present a list of the Simuliidae collected in winter and summer drift samples from the Neste d'Aure (altitude $1100-1600 \mathrm{~m}$ ). The population distribution was analysed using data from eight drift stations chosen upstream and downst ream of three reservoirs. The importance of a flow reserve below a dam is emphasised.

\section{Introduction}

L'étude de la faune macroinvertébrée du réseau hydrographique de la Neste d'Aure a été entreprise ces dernières années en vue de compléter les connaissances acquises sur la distribution altitudinale des espèces. Des recherches sur l'ensemble de cette faune avaient été réalisées antérieurement dans un premier intervalle d'altitude situé dans la partie haute du réseau, à l'étage alpin et subalpin: 1800-2500 m, torrent de l'Estaragne (Lavandier 1979). Il était particulièrement intéressant de poursuivre ces investigations à l'étage montagnard, collinéén et en basse vallée afin de déterminer les carac. téristiques des différents peuplements. Dans un deuxième intervalle d'altitude, $1100-1600 \mathrm{~m}$, Gazagnes (1983) a poursuivi la détermination de l'ensemble de la faune. Un troisième volet d'altitude 425-1100 m, traitera prochainement, après une étude

I. Laboratoire d'Hydrobiologie, UA CNRS 695, Université Paul Sabatier, 118 route de Narbonne, 31062 Toulouse Cedex, France. préliminaire (Bouguenec \& al. 1985), de la faune de la basse vallée, complétant ainsi la répartition altitudinale des es pèces macroinvertébrées dans la vallée d'Aure.

Issue du travail cité plus haut (Gazagnes 1983), la présente note concerne les Simuliidae, Diptères Nématocères peuplant les eaux courantes à l'état immature. Depuis 1950, quelques auteurs ont largement contribué à l'amélioration de la connaissance de cette famille : Grenier (1949-1953) pour la taxonomie et la biologie, Dorier (1962-1963) et Giudicelli (1968) pour l'écologie. Par la suite, les Simulies ont fait parfois l'objet d'études faunistiques dans le cadre de travaux pour connaitre de façon précise la faune des rivières. Réputées de détermination "difficile ", elles sont encore un peu négligées, notamment dans les travaux étudiant les répercussions engendrées par l'installation d'ouvrages hydrauliques ou hydroélectriques sur la faune benthique des torrents. Les auteurs leur préfèrent les groupes plus connus tels que : Chironomides, Plécoptères... Avec les nombreuses listes faunistiques données pour ces groupes, on ne trouve la plupart du temps que "Simuliide" 
pour cette famille, celle-ci étant prise en compte dans son ensemble, ou pas du tout! Une autre raison de cette " mise à l'écart "est l'imperfection de la nomenclature. Depuis quelques années cependant, celle-ci s'est amélioréc grâce aux recherches de Zwick (1974) et à l'adoption de la classification de Crosskey (1981) par les spécialistes de plusieurs pays européens : cette dernière regroupe provisoirement les sous-genres par ordre alphabétique.

Durant les stades immatures, cette famille constitue cependant une partie non négligeable de la faune benthique. Excellents indicateurs biologiques, ces animaux sont des formes dérivantes capturées dans des biotopes où les vitesses sont maximales; or, la zone étudiée possédant des tronçons à débit modifié par l'existence de trois retenues, nous avons été amenés à analyser les différences existant entre les peuplements simuliidiens des tronçons de rivière à débit naturel (armont des retenues) et ceux des tronçons aménagés à débit réservé ou non (en aval des prises d'ealu). La comparaison entre les différentes populations nous donnera des indications précieu. ses pour montrer l'importance des débits réservés.

Dans les Pyrénées, Chauvet (1983) a déjà posé le problème de l'influence d'une réduction de débit sur les Invertébrés et les Salmonidés d'un torrent ariègeois : l'Aston; il a souligné l'importance de la régularité d'un débit réservé. L'impact des barrages sur la faune a été étudié dans d'autres régions françaises: dans le sud-ouest sur le Lot, la Dordogne et la Truyère (Décamps et al. 1979) et dans les Alpes de Haute-Provence sur le Verdon (Grégoire 1981).

D'autre part, les conséquences de l'implantation de barrages sur le zoobenthos, ont été exposées à la même époque dans les conclusions du Symposium organisé sur "l'écologie des fleuves régulés "(Ward et Stanford 1979). S'il y est dit que les Invertébrés résistant le mieux aux modifications de débit à l'aval des barrages sont les Chironomides, les Amphipodes et lęs Olighochètes, on y parle peu des Simuliess. Raadstadt (1983) étudie pour la première fois l'impact d'un barrage en Norvège à l'aide des Simuliidae ; il note une densité maximum (160000 individus $/ \mathrm{m}^{2}$ ) dans les stations déversoirs, représentant 80-90\% de la faune, alors qu'en aval la densité fléchit brusquement au profit des Chironomides.

\section{2. - Méthodes et stations d'étude}

\section{1. - Méthodes}

Trois séries d'échantillonnage ont été effectuées en août 1980 , septembre 1980 et février 1981 .

Les échantillons ont été prélevés toutes les trois heures durant un cycle de $24 \mathrm{~h}$.

Composé essentiellement de larves, d'exuvies nymphales et de quelques nymphes, le matériel a été récolté à l'aide d'un filet de dérive simple. Ce dernier est composé d'une buse carrée de $12,5 \mathrm{~cm}$ de côté, prolongée de deux filets emboités : le premier, simple treillis platifie à grosses mailles $(1 \mathrm{~cm})$, est destiné à arrêter les brindilles et les feuilles mortes afin d'éviter un colmatage trop rapide: le deuxième (longueur $120 \mathrm{~cm}$, vide de maille $240 \mu$ ) retient les organismes de la dérive. Les filets étaient entièrement immergés dans des zones à fort débit, où la profondeur ne dépassait pas celle du filet, de manière à filtrer toute la colonne d'eau.

\subsection{Stations d'étude}

Huit stations à débit naturel, modifié ou réservé furent choisies et suivies régulièrement (fig. 1):

1: Neste de la Géla, en amont de la retenue de prise d'eau:

2: Neste du Badet, en amont de la retenue de prise d'eau :

3: Neste de la Géla, en aval de la retenue;

4: Neste du Badet, en aval de la retenue ;

5: Neste d'Aragnouet:

6: Neste de Couplan ;

7: Neste d'Aure, en amont de la retenue de Fabian ;

8: Neste d'Aure, en aval de la retenue de Fabian.

Les Nestes ont un régime torrentiel, de type nival à nival de transition : fonte des neiges en mai-juin et pluies en octobre-novembre.

Les principales caractéristiques physiques des stations sont résumées dans le tableau 1 . Pour plus de détails sur les autres caractéristiques physico. chimiques, on pourra se reporter au travail de Gazagnes (i983).

Les stations 1 et 2 étant situées à l'amont des retenues ( $f i g .1)$, leurs débits sont naturels. Situées par contre en aval, les stations $3,4,5$ ne possèdent pas de débit régulé ou réservé: l'eau qui s'écoule aux étiages provient uniquement des petits ruisselets du 


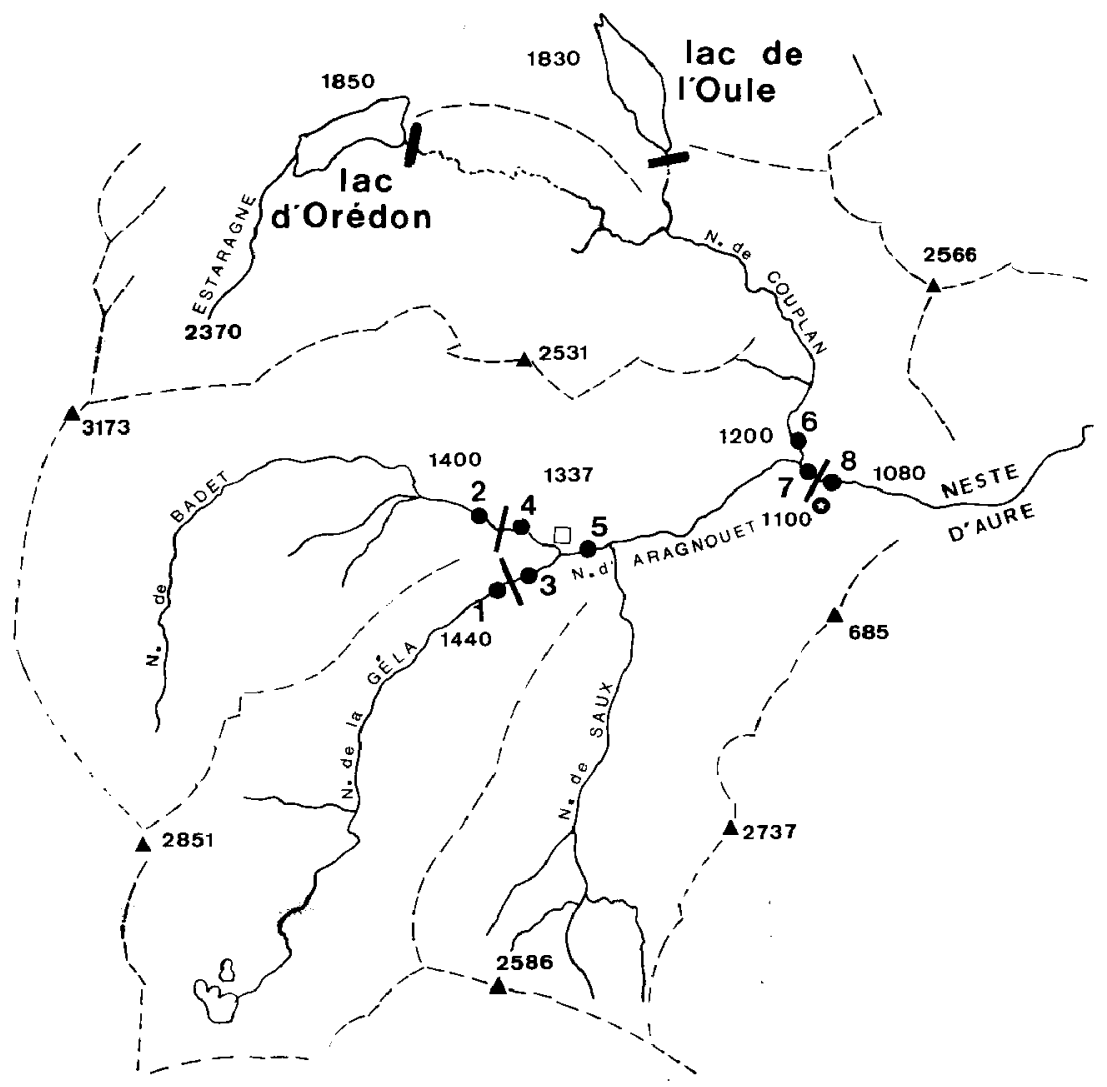

Fig. 1: Réseau hydrographique de la Haute Neste d'Aure.

1 à 8 : stations d'études.

trait fin : prises d'eau,

trait épais : barrages,

étoile blanche entourée de noir: Fabian,

carré blanc : Le Plan. 
Tableau 1. Principales caractéristiques physiques des stations A : Août, S: Septembre, F : Février.

\begin{tabular}{|c|c|c|c|c|c|c|c|c|c|c|c|}
\hline \multirow{2}{*}{$\begin{array}{c}\mathrm{N}^{\circ} \\
\text { Stations }\end{array}$} & \multirow{2}{*}{$\begin{array}{l}\text { Altitude } \\
\text { (In) }\end{array}$} & \multirow{2}{*}{$\begin{array}{l}\text { Nature du } \\
\text { débit }\end{array}$} & \multicolumn{2}{|c|}{$\begin{array}{l}\text { Débit moyen } \\
\qquad\left(\mathrm{m}^{3 / \mathrm{s}}\right)\end{array}$} & \multicolumn{2}{|c|}{$\begin{array}{l}\text { Profondeur } \\
\text { maxi }(m)\end{array}$} & \multicolumn{2}{|c|}{$\begin{array}{l}\text { Largeur } \\
\text { maxi (m) }\end{array}$} & \multicolumn{3}{|c|}{$\begin{array}{c}\text { Température de } \\
\text { l'eau }\end{array}$} \\
\hline & & & Etiage & Crue & Etiage & Crue & Etiage & Crue & $\mathrm{A}$ & $\mathrm{s}$ & $\bar{F}$ \\
\hline \multicolumn{12}{|c|}{ Etage montagnard } \\
\hline 1 & 1440 & naturel & 0,3 & 2.4 & 0.35 & 0,8 & 4 & 5 & $9^{\circ}$ & $8^{\circ} 6$ & \multirow{3}{*}{$\begin{array}{c}4^{\circ} 5 \\
\text { Retenut } \\
2 \quad 1^{\circ} 6\end{array}$} \\
\hline 2 & 1400 & naturel & 0,5 & 4,4 & 0,26 & 0,7 & 8 & 12 & $9^{\circ} 4$ & $8^{\circ} 9$ & \\
\hline 3 & 1360 & $\begin{array}{c}\text { débit perturbé, } \\
\text { non réservé. }\end{array}$ & 0,004 & 0,4 & 0,1 & 0,55 & 0.5 & 4 & $12^{\circ} 2$ & $11^{\circ} 2$ & \\
\hline 4 & 1360 & \multirow[b]{2}{*}{$"$} & 0,011 & 0,9 & 0,15 & 0,45 & 1,25 & 4,5 & $14^{\circ} 1$ & $12^{\circ} 7$ & $2^{\circ}$ \\
\hline 5 & 1360 & & 0,015 & 1,3 & 0.15 & 0,35 & 1 & 7 & $14^{\circ}$ & $11^{\circ} 8$ & $1{ }^{\circ} 8$ \\
\hline \multicolumn{2}{|c|}{ Etage collinéen } & & & & & & & & & & \\
\hline 6 & 1200 & \multirow{3}{*}{$\begin{array}{l}\text { naturel } \\
\text { modifié } \\
\text { naturel } \\
\text { modifié } \\
\text { réservé }\end{array}$} & 0,3 & 1,1 & 0,4 & 0,5 & 6 & 6,5 & $12^{\circ} 8$ & $11^{\circ} 6$ & $2^{\circ} 3$ \\
\hline 7 & 1100 & & 0.4 & 1,9 & 0.25 & 0.65 & 4,5 & 9 & $12^{\circ} 2$ & \multirow{2}{*}{\multicolumn{2}{|c|}{${ }_{11^{\circ} 3} \begin{array}{c}2 \circ 6 \\
\text { Retenuc }\end{array} 2^{\circ} 5$}} \\
\hline 8 & 1080 & & 0,06 & 1,25 & 0,2 & 0,45 & 1,6 & 3,5 & $11^{\circ} 9$ & & \\
\hline
\end{tabular}

bassin versant et, pour une plus faible part, des fuites de vannes des prises d'eau ; les débits sont alors très faibles. Le débit de la station 6 provient principalement des sources d'Artigusse (1590 m), les eaux originelles étant captées plus haut par les barrages du Massif du Néouvielle. La station 7 reçoit les eaux de la station précédente à débit assez constant et de la Neste d'Aragnouet, à faible débit d'étiage. A l'aval de la retenue de Fabian, la station 8 est la première station à bénéficier d'un débit réservé (125 1/s).

Toutes les stations en aval des retenues $(3,4,5$ et 8) peuvent retrouver leurs débits d'origine au moment des crues et s'uniformiser sur ceux des autres stations.

A cause de leur très faible débit en période d'étiage, les eaux des stations 3, 4 et 5 subissent une élévation de température, une forte minéralisation et un léger accroissement de certaines composantes physico-chimiques.

Le substrat est le même sur l'ensemble du réseau : il est composé de galets, de blocs et de graviers. L'analyse des sédiments a montré deux types de dépôt à des périodes différentes. Des graviers et des sables grossiers se déposent régulièrement dans les stations 1, 2, 6 et 7 puis dans les autres stations quand il y a crue ou lâchure (régime torrentiel). Au contraire, les matériaux fins s'accumulent dans les retenues ou en aval de celles-ci lorsque la vitesse du courant diminue (st. 3, 4 et 5 en étiage) et colmatent les interstices par décantation.

\section{3. - Peuplement faunistique des Simuliidae de la Haute Neste d'Aure}

La liste faunistique des espèces récoltées par dérive est récapitulée dans le tableau II. Elle suit la classification de Crosskey (1981).

16 taxons ont été déterminés dont 13 identifiés à l'espèce. Dans chaque échantillonnage de dérive, ont été récoltées des exuvies nymphales et des larves en nombre particulièrement important, comparé aux autres familles (Gazagnes 1983, Gazagnes \& Laville 1985), et des nymphes en très petite quantité. L'analyse de la répartition s'appuie surtout sur les larves.

En tant que dérive inerte, les exuvies nymphales nous permettent de situer facilement les prinçipales espèces émergeant au moment de la capture. Par exemple, nous pouvons déduire qu'au mois d'août émergent à cet te altitude : $P$.(P.) rufipes, $S$.(S.) argenteostriatum, S.(S.) argyreatum, S.(S.) monticola, et $S .(T$.) bezzii. En septembre, le groupe monticola continue ses émergences avec S.(O.) auricoma. La 
Tableau II : La dérive des Simuliidae par especes et par 24 h.

A : Août, $\mathbf{S}$ : Septembre, F : Février.

\begin{tabular}{|c|c|c|c|c|c|c|c|c|c|c|c|c|c|c|c|c|c|c|c|c|}
\hline & \multirow{2}{*}{\multicolumn{2}{|c|}{1}} & \multirow{2}{*}{\multicolumn{2}{|c|}{2}} & \multirow{2}{*}{\multicolumn{2}{|c|}{3}} & \multirow{2}{*}{\multicolumn{3}{|c|}{4}} & \multirow{2}{*}{\multicolumn{2}{|c|}{ s }} & \multirow{2}{*}{\multicolumn{3}{|c|}{6}} & & & & & & \\
\hline & & & & & & & & & & & & & & & \multicolumn{2}{|l|}{$?$} & \multicolumn{3}{|c|}{8} & \\
\hline & $A$ & 5 & $A$ & $\overline{s \quad 1}$ & $\mathrm{~s}$ & $\bar{F}$ & 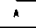 & $\mathbf{s}$ & $\bar{f}$ & $A S$ & $\bar{s}$ & $A$ & s & $\bar{F}$ & $A$ & $\mathrm{~F}$ & $A$ & 5 & $\bar{f}$ & \\
\hline Metacnephia sp. Cross. & 5 & & & & & & & & & & & 1 & & & & & & & & $=$ \\
\hline Erosimulium (Prosimulium) hirtipes fr. & & & & & & 17 & & & 20 & & 1 & & & 17 & & & & & 6 & 61 \\
\hline p. (p.) rufipes mg. & & & & & & & & & & & & & & & 1 & & & & & 1 \\
\hline simulium (Eusimulium) gr. aureum & & & & & & & & & & & & & . & & 3 & & 3 & & & 6 \\
\hline 5. (Nevermangia) brevidens Rubz. & 4 & & & & & & & & & & & & & & & & & & & 4 \\
\hline s. (N.) carthusiense Gr. D Dor. & 26 & & 20 & 12 & & & & & & & & 3 & 6 & 1 & 69 & & 74 & & & 213 \\
\hline S. (N.) eryophilum Rubz. & 2 & 1 & 16 & & & & 35 & & 5 & & 1 & 13 & & 5 & 183 & & 26 & & & 2 a? \\
\hline s. (N. ) gr. vernum. & & & & & & & & & & & & 17 & & & & & 4 & & & 21 \\
\hline$\underline{s}$. (Öbuchovia) auricona $\mathrm{Mg}$. & 1 & 1 & & & & & & & & & & 13 & & 24 & 20 & 1 & 2 & & 3 & 65 \\
\hline s. (odagmia) nitidifrons Edw. & & & 2 & & & & & & & & & & & & 1 & & & & & 3 \\
\hline $\overrightarrow{\text { s. }}$ (Simuliun $)$ argenteostriatum str. & 30 & & 1 & & & & & & & & 1 & 14 & & & 15 & & 6 & & & 67 \\
\hline$\vec{s} . \overline{\left.s_{+}\right)}$argyreatum $\mathrm{Mg}$. & 30 & 79 & 16 & 847 & 11 & 2 & 16 & 155 & & 8 & 14 & 1205 & 339 & 111 & 2296 & 11 & 4221 & 138 & 87 & 8830 \\
\hline s. (s.) monticola fried. & & & & & & & & & & & & & & & & & & & & \\
\hline s. (s.s.) variegatum Mg. & & 22 & 1 & 1 & & & & & & & & 349 & 5 & & 783 & & 311 & & & 1478 \\
\hline s. (S.) tuberosum Lunds. & & l & & & & & & 1 & & & & & & & & & & & & 2 \\
\hline s. (Tetisinulium) bezii c. & & & & & & & & & & & & 56 & 3 & & GH & & $j \vdots$ & & & 158 \\
\hline & & & 56 & 97 & 011 & 19 & 5. & 156 & 25 & 08 & 17 & 1673 & 353 & 158 & 3459 & 12 & 4658 & 138 & 96 & 11196 \\
\hline
\end{tabular}

quantité d'exuvięs recueilliẹs dans le filet peut donner une indication sur le pourcentage d'individus en émergence par espèce.

La dérive vivante est constituée par des larves immatures pour la plus grande partie, les autres ne dérivant qu'en nombre très restreint.

Toutes les espèces, sans exception, sont rhéophiles. A cette altitude, sont surtout recueillies celles à large répartition altitudinale: : $P,(P$.$) rufipes, S .(N)$ carthusiense, S.(N.) cryophilum, S.(S.) argenteostriatum, et les deux especes du groupe monticola. Toutes sont recensées par Lavandier (1979) entre 1800 et $2500 \mathrm{~m}$.

En second lieu, on trouve des espèces de basse ou moyenne altitude, qui atteignent ici leur limite supérieure de répartition: $P$.(P.) hirtipes, $S$.(S.) variegatum, S.(T.) bezzii.

De vaste répartition altitudinale $300-2500 \mathrm{~m}, P$. $(P$.) rufipes est sténotherme d'eau froide et montagnarde. Lavandier l'a signalée en haute altitude $(1976,1979)$. Nous l'avons recensée au mois d'août (st. 7) grâce aux exuvies nymphales.

- Un peu moins sténotherme que la précédente, $P$.(P.) hirtipes atteint ici sa limite de fréquence altitudinale puisqu'elle n'est présente que dans les stations 3 à 8 en février. Son maintien dans les stations 3 à 5 est sans doute dû à la crue de printemps qui lui permettrait de se nymphoser avant la forte réduction de débit estivale.

- En ce qui concerne l'espèce du genre Metacnephia, nous préférons ne donner aucune précision, les taxa étant actuellement en révision (H. Zwick).

- S.(N.) brevidens se trouve dans les ruisseaux et les déversoirs de l'étage montagnard ; l'espèce avait déjà été signalée par Lavandier (1979), en haute montagne, avec de très faibles effectifs. Elle devrait atteindre ici sa limite inférieure de distribution puisqu'on la trouve seulement dans la station 1, en très petit nombre également (4 larves).

- S.(N.) carthusiense : de vaste répartition altitudinale et sténotherme d'eau froide, cette espèce n'est pas présente dans le tronçon sans débit réservé, sans doute à cause de l'élévation de température estivale. Elle se retrouve dans les st. 6 à 8 avec un effectif réduit, alors qu'elle domine toutes les autres espèces dans le torrent d'Esta ragne d'amplitude thermi. que : $5^{\circ}-11^{\circ}$ (Lavandier 1979). Par contre, dans une autre vallée proche : lac de l'Isle et son déversoir, Estibère $2100 \mathrm{~m}$, l'espèce est dominée largement par S.(S.) argyreatum et $S$.(N.) cryophilum : amplitude thermique obtenue par dérive sur $24 \mathrm{~h}: 10^{\circ}-16^{\circ}$ en 
juillet 1974 (Thomas 1981). Cette différence s'explique par une température estivale nettement plus élevée dans la cuvette des lacs d'Estibère orientée au sud. Le même résultat a été obtenu par Gonzàles (1980) dans les torrents andorrans.

-S.(N.) cryophilum : plus eurytherme que la précédente, est présente à toutes les stations, en faible effectif.

- Les quelques individus de l'espèce du gr. aureum se cantonnent dans les deux dernières stations. A cause de la petitesse des larves et de la difficulté à reconnaître les espèces du groupe à l'état larvaire. nous les désignerons sous le nom de $S$.(E.) $g r$. aureum.

- Les deux espèces du groupe monticola: S.(S.) argyreatum et $S$.(S.) monticola, bien différenciables à l'état nymphal et chez la larve mature, ne peuvent être séparées convenablement sur les très nombreuses petites larves de la dérive. Elles ont été par conséquent réunies sous le nom de $S$.(S.) gr. monticola, afin de les comptabiliser, tout en sachant, par l'examen des larves âgées et des exuvies nymphales, que S.(S.) argyreatum est de loin la plus fréquente.

Cette dernière est présente à tous les niveaux alors que S.(S.) monticola, à population bien moins dense, n'est présente qu'épisodiquement dans les biotopes étudiés. A ce niveau altitudinal, l'immense majorité des larves de Simuliidae $(78 \%)$ appartient au gr. monticola et par conséquent à l'espèce $S$.(S.) argyreatum.

- Très peu représentée dans l'Estaragne $(1800-2500 \mathrm{~m})$, S.(S.) argenteostriatum est connue également pour sa vaste répartition altitudinale. De faible effect if au niveau de la haute Neste, elle est aussi bien présente dans les stations à débit naturel (1-2-6-7) que dans celle à débit réservé (St .8), mais absente des stations sans débit réservé.

- S.(S.) variegatum a l'effectif le mieux représenté $(13 \%)$ après le gr. monticola. Espèce de basse altitude, elle atteint ici sa limite supérieure de répartition. Bien qu'eurytherme, elle recherche malgré tout les eaux fraîches et, pour cette raison, est absente en août des st. 3, 4 et 5 .

- $S .(T$.) bezzii est souvent associée à l'espèce précédente, et atteint ici sa limite supérieure altitudinale. Eurytherme, elle ne dépasse pas les st. 6,7 et 8 où elle est encore moyennement représenté, mais ne s'accommode pas du régime d'étiage, sans courant rapide, des st. 3 à 5.
- S.(O.) auricoma, très rhéophile, est commune dans les Alpes à faible altitude (Dorier 1962) où elle préfère les torrents à fond stable (dalles ou blocs). Dans nos régions, il semblerait qu'elle at teigne des altitudes bien plus élevées puisque Lavandier l'a capturée au-delà de $2000 \mathrm{~m}$. Absente, comme les trois précédentes, des st. 3 à 5 . l'espèce n'a que de faibles effectifs en août dans les st. 6 à 8 , avec surtout des exuvies nymphales; sa présence est très sporadique dans la st. 1.

Des huit espèces recensées par Lavandicr (1979), seule $P$. $(P$.$) latimucro ne figure pas dans nos prélè-$ vements. Cette espèce plus psychrotherme n'est connue dans nos régions qu'en haute altitude.

En ce qui concerne les données préliminaires fournies par Lavandier en 1976, nous retrouvons sensiblement les mèmes espèces dans les mêmes tranches d'altitudes (voir fig. 2 Lavandier, 1976). Ce sont des espèces, d'une part, de grande répartition altitudinale, comme S.(N.) carthusiense, S.(N.) cryophilum, le gr. monticola, S.(S.) argenteost riatum... etc... ; d'autre part, de basse ou moyenne altitude qui at teignent ici leur limite supérieure de répartition, comme S.(S.) variegatum, S.(T.) bezzii.

\section{4. - Analyse du peuplement simu liidien}

Une constante diminution du nombre d'exuvies nymphales et de l'effectif larvaire dans les dérives a été constatée du mois d'août au mois de février. Par station d'étude, on obtient les résultats suivants pour les larves.

- aux st. 1 et 2, en amont des retenues où le débit est naturel, les Simulies réprésentent $8 \%$ des larves de Diptères en août, $15 \%$ en septembre; en février, il n'y a pratiquement pas de Simulies dans les dérives.

- aux st. 3, 4 et 5, le nombre d'individus dérivants est très faible.

- à la st. 6, la dérive des Simulies représente en août $64 \%$ de celle de tous les Diptères, $44 \%$ en septembre et $15 \%$ seulement en février alors que les Chironomides dominent à cette époque.

- à la st. 7, en août, on a dénombré $68 \%$ de larves de Simulies et à la st. 8 (débit réservé), elles 
représentent $81 \%$ de tous les Diptères. Par contre en septembre et surtout en février, les Simulies sont largement dominées par les Chironomides.

Deux espèces sont dominantes dans la haute Neste: S.(S.) argyreatum ( 8830 individus pour le gr. monticola) et $S$.(S.) variegalum (1478 ind.). Trois especes peuvent être considérées comme subdominantes: $S$.(N.) carthusiense, $S$.(N.) cryophilum et $S .(T$.) bezzit et trois autres, fréquentes: $P .(P$.) hirtipes, $S .(O$.) auricoma et $S$.(S.) argenteostriatum.

Le régime nival des eaux que fréquente la faune sténotherme d'eau froide est représenté par la Géla (st.1) et le Badet (st.2); il se retrouve également en aval des retenues (st. 3 a 5) pendant la crue de printemps, crue prolongée (avril à juillet) qui provient des sources froides de l'étage alpin et subalpin.

Au niveau des st .6, 7 et 8 , on découvre une faune plus eurytherme. Situées à une altitude plus basse et favorisées par une crue de printemps plus brève. les sources d'Artigusse sont à l'étage montagnard -, ces stations subissent un réchauffement des eaux nlus rapide $\left(11,9^{\circ} \mathrm{C}\right.$ à $12,8^{\circ} \mathrm{C}$ en août) et jouissent d'un substrat plus stable. Les espèces de basse et moyenne vallée comme $S$. $(T$.) bezzii, $S .(S$.) variegatum, peuvent par conséquent coloniser les Nestes de Couplan (st.6) et d'Aure (st.7 et 8 ) dont le régime des eaux, à ce niveau, est de type pluvio-nival.

L'influence des aménagements hydroélectriques se fait sentir par conséquent sur deux types de faune : sténotherme aux stations 3 à 5 et eurytherme à la station 8 .

Tableau III : Nombre d'espèces par station

\begin{tabular}{crccc}
\hline Dates & $\begin{array}{c}\text { Août } \\
1980\end{array}$ & $\begin{array}{c}\text { Septembre } \\
1980\end{array}$ & $\begin{array}{c}\text { Février } \\
1981\end{array}$ & $\begin{array}{c}\text { Total des } \\
\text { espéces }\end{array}$ \\
\hline Stations & & & & \\
1 & 7 & 5 & & 9 \\
2 & 6 & 3 & 1 & 6 \\
3 & 0 & 1 & 2 & 2 \\
4 & 2 & 2 & 2 & 4 \\
5 & 0 & 1 & 4 & 4 \\
6 & 9 & 4 & 5 & 10 \\
7 & 10 & & 2 & 10 \\
8 & 9 & 1 & 3 & 10 \\
\hline
\end{tabular}

Il existe une diversité spécifique importante dans les peuplements naturels: st. 1, 2 et 6,7 . (tableau III). La réduction des débits provenant des aménagements hydroélectriques, exerce une influence différente sur des peuplements originels distincts: nous dirons qu'à la st.8 (débit réservé) la faune eurytherme est maintenue en état en ce qui concerne notre groupe, puisque $81 \%$ de la faune dérivante de Diptères sont des Simulies, en progression par rapport aux st. 6 et 7 , situées en amont de la retenue.

Par contre aux st.3, 4 et 5 (débits perturbés), les conclusions sont différentes; la faiblesse du débit diminue à la fois la densité et la richesse du peuplement sténotherme. De 9 espèces dans la st.1, (amont de la retenue de la prise d'eau sur la Géla), on passe à 2 espèces dans la st.3 (aval de la retenue).

\section{AFFINITESS ENTRE LES STATIONS}

Afin d'évaluer les ressemblances entre les populations des différentes stations, nous avons comparé deux à deux ces peuplements, à l'aide de l'indice de Sørensen (1948 (Tableau IV)

IS $=\frac{2 \mathrm{c}}{\mathrm{a}+\mathrm{b}}$

avec $c=$ nombre d'espèces communes aux 2 stations.

$\mathrm{a}$ et $\mathrm{b}=$ nombre d'espèces de chaque station.

Ces données feront l'objet d'un travail plus élaboré de comparaison avec les populations de la basse vallée d'Aure d'une part, et avec celles de la vallée d'Ossau (Pyrénées atlantiques) d'autre part.

L'affinité entre les st. 1 et 2 est assez bonne, toutes deux étant situées à l'amont des retenues (débit naturel). De mèrne, entre les stations à débit naturel 1 et 2 ou naturel modifié 6 et 7 .

L'affinité entre les stations 6,7 et 8 est très forte : ce sont les plus affines; elles possèdent une grande richesse spécifique avec 10 espèces chacune. Le débit réservé de la station 8 maintient donc une bonne diversité spécifique, de manière analogue à celle des Chironomides et des Ephéméroptères.

\section{5. - Discussion et conclusion}

L'étude de la dérive des Simuliidae dans les huit stations situées en amont ou en aval d'ouvrages 
Tableau IV. Similarité faunistique des populations des différentes stations deux à deux (Indice de S $\phi$ rensen. 1948)

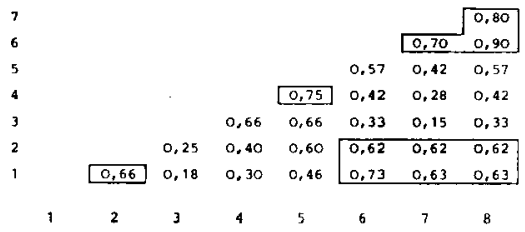

hydroélectriques sur la Haute Neste d'Aure nous a permis :

1) de déterminer les espèces présentes à cette altitude.

Toutes sont rhéophiles, fréquentant des cours d'eau rapides, à fond nu de végétation. Sténothermes d'eau froide en majorité, et déjà recensées à un niveau supérieur $(1800-2500 \mathrm{~m})$, elles sc ict rouvent ici, soit qu'elles possèdent une vaste répartition altitudinale (P.(P.) rufipes, $S$.(N.) carthusiense, $S$.(N.) cryophilum...), soit qu'elles arrivent à leur limite inférieure de distribution ( $S$.(N.) brevidens). D'autres espèces plus eurythermes arrivent à leur limite supérieure altitudinale : $S$.(S.) variegatum, $S$.(T.) bezzii. Le peuplement peut donc être considéré comme étant intermédiaire : " torrent de transition", entre un torrent de haute montagne et une rivière de moyenne montagne.

2) de constater que :

a) la présence d'un débit réservé à l'aval d'une retenue (station 8) maintient la faune simuliidienne en état. $81 \%$ des larves de Diptères dérivantes sont des Simulies, elles atteignent ici leur dérive la plus forte (4 892 individus sur 24 h). Les stations 6, 7 et 8 sont les plus affines. Ce peuplement, dense et riche en espèces, paraît lié à un débit régulier surtout lors des étiages.

b) la présence d'un débit très perturbé et non réservé à l'aval des prises d'eau du Badet et de la Géla ne maintient pas la faune en état; il n'existe plus de bonne diversification ni d'abondance du peuplement : en août, on assiste même à sa disparition complète (st. 3 et 5). Cet appauvrissement en individus et en espèces provient de la faiblesse du débit à l'étiage (Tableau I) qui occasionne un réchauffement des eaux et une forte minéralisation (passage d'un milieu lotique à un milieu lentique entre autre). Ces variations de débit détruisent par conséquent des éléments importants d'un biotope en courant rapide et de la faune benthique rhéophile. C'est la conclusion à laquelle sont arrivés $\mathrm{d}$. leur côté : Fischer \& Lavoy (1972), Trotsky \& Gregory (1974) dans leur étude sur la « Upper Kennebec river " et plus récemment Gazagnes \& Laville (1985). pour les Chironomides de la Haute-Neste d'Aure.

Dans ce torrent, le même phénomène s'observe chez les Ephéméroptères, les Plécoptères et les Trichoptères. Peut-être à cause de la disparition de ces groupes et malgré leur diminution en espèces, les Chironomides restent ainsi prédominants dans ces stations.

c) la crue de printemps permet cependant à cer taines espèces sténothermes, dont la nymphose $i n$ relativement précoce, de se maintenir dans les sti: tions 3 à 5 (P.(P.) hirtipes).

\section{Travaux cltés}

Bouglienec (V.). Meurgues (S.) \& Sourzac (C.) 1985. Introduction à l'étude d'une rivière pyrénéenne aménagée : la Neste d'Aure. D.E.A Hydrobiologie, Universilé Paul Sabatier. Toulouse.

Chauvet (E.) 1983. Influence d'une réduction de débit sur un tor. rent de montagne : l'Aston (Ariege). Annls Limmol, 19(1) : 45-49.

Crosskey (R.-W.) 1981. Simulid Taxonomy. The contemporary scene. Blackflies : 3.18. In : The future of biological methods in integrated control. Laid M., ed. Academic press. London. 399 p.

Decamps (H.), Capblancq (J.), Casanova (H.) \& Toureng (J.-N.) 1979 Hydrobiology of some regulated rivers in the South-West of France p. 273-288. In : J.W. Ward and J.-A. Stanford (E.d.) The ecology of regulated streams. N.Y.

Dorier (A.) 1962-1963. Documents pour servir à la connaissance dt." Simuliidae du Sud-Est de la France. Trav. Lab. Hydrob. Pin Grenoble, : 54-55: 7-79.

Fischer (S.G.) \& Lavoy (A.) 1972. Differences in littoral fauna dut to fluctuating water levels below a hydroelectric dam. J. Fish Res. Bd Can , $29: 1472.1476$.

Gazagnes (G.) 1983. Contribution à l'étude de la dérive des Inver. tébrés sur la Haute Ncste d'Aure : impact des amenagements hydruélectriques. Thèse 3eme cycle, Hydrobiologie, Université Paul Sabatier. Toulouse (France) : 194 p. +1 annexe.

Gazagnes (G.) \& Laville (H.) 1985. Etude faunistique des chironomidés (Diptera) de la Haute+Neste d'Aure (Pyrénées Centrales) : impact des aménagements hydroélectriques. Anmls Limnol. 21(2): 149.159.

Giudicelli (J.) 1968. Recherches sur le peuplement, J'écologie et la biogéographie d'un réscau hydrographique de la Corse centrale. Thèse Doctorat ès sciences naturelles, Université AixMatseille, $437 \mathrm{p}$.

González (G.) 1980. Primeres dades sobre la distribucio dels Simulitidae (Diptera, Nematocera) d'Andorra, Bull. Inst. Cat. Hist. Nar., (sec. Zool)., 45, (3) : 97.106. 
Grégoire (A.) 1981. Contribution à l'étude d'une rivière aménagée te Verdon (Alpes de Haute Provence). Les lacs de barrage et les tronçons de cours d'eau à débit régulé. These Doctorat es sciences naturelles. Université Aix-Marseille. I : $213 \mathrm{p}$

Grenier (P.) 1949. Contribution à l'étude biologique des Simuliidès de France. Physiol.comp. Oecol. 1 : 165-330.

Grenier (P.) 1953. Simuliidae de France et d'Afrique du Nord. Ency clop. Ent. Ser. A. Paris, 29:1.170.

Latvandier (P.) 1976. Premières données sur la répartition des Simu. liidae (Dipteres. Nématocères) dans la vallèe d'Aure (Hautes Pyrénées, France). Bull. Soc. Hist. Nat. Toulouse., 112 (1-2) 89.95 .

I.avandier (P.) 1979. Ecologie d'un torrent pyrénéen de haute alti. tude : l'Estaragne. Thèse Doctorat ès sciences naturelles, Université Paul Sabatier. Toulouse, $532 \mathrm{p}$.

Raadstadt (J.) 1983. The effect of weirs on macrobenthos in regu. lated rivers, with special emphasis on blackflies (Diptera, Simu liidae). Terkelprojektel, Nonvège, $23: 6.94$.

syrensen (T.) 1948. A method of establishing groups of equal amplitude in plant sociology based on similitary of species content and its application to analyses of the vegetation on Danish com. mons. Biol. Skr., 5 : 1-34.
Thomas (A.) 1981. Travaux sur la taxonomie, la biologie et l'écologie d'Insectes torrenticoles' du Sud-Ouest de la France (Ephéméroptères et Diptères : Dixidae, Cecidomyidae, Rhagionidae et Athe ricidael, avec quelques exemples de perturbations par I'homme. Thèse Doctorat ès sciences nalurelles, Université Paul Sabatier. Toulouse. $330 \mathrm{p}$.

Troszky (H.M.) \& Gregory (R.-M.) 1974. The effect of water flow manipulation below a hydroelectric power dam on the botton fauna of the Upper Kennebec River, Maine. Trans. Am. Fisch. Soc., $103: 318-324$.

Ward (J.V.) \& Stanford (J.-A.) 1979. Symposium summary and conclusions : pp 377-385. In : Ward (J.-V.) \& Stanford (J.-A.) (Ed) The ecology of regulated streams, New York.

Zwick (H.) 1974. Faunistisch-ökologische und taxonomische Untersuchungen an Simuliidae (Diptera), unter besonderer Berücksichtigung der Arten des Fulda-Gebietes. Abhandl Senckenberg. Naturf. Gessellschaft. $533: 1-116$. 